\title{
The use of ultrasound in inflammatory bowel disease
}

\author{
Torsten Kucharzik, Klaus Kannengiesser, Frauke Petersen
}

Lüneburg Hospital, University of Hamburg, Germany

Abstract

Imaging in inflammatory bowel disease (IBD) plays a pivotal role in the primary diagnosis, as well as during the management of patients with known IBD. The evolution of ultrasound equipment and the growing expertise of examiners have both enhanced the role of intestinal ultrasound in the assessment of the gastrointestinal tract in IBD patients. Intestinal ultrasound has been shown to have high sensitivity and specificity, as well as high positive and negative predictive value, in the detection or exclusion of intestinal inflammatory activity in IBD. The obvious advantages of intestinal ultrasound over other imaging modalities include non-invasiveness, rapid availability and low costs. This review summarizes the current developments in the use of intestinal ultrasound for the detection of IBD and its complications, and discusses its use in the management of patients with IBD. Indications for the use of intestinal ultrasound in daily practice are presented, expanded by new developments such as contrast-enhanced ultrasonography and elastography.

Keywords Intestinal ultrasound, inflammatory bowel disease, contrast-enhanced ultrasound

Ann Gastroenterol 2017; 30 (1): 1-10

\section{Introduction}

Intestinal ultrasound (IUS) plays an important role in the recognition, diagnosis, and monitoring of many gastroenterological diseases and is becoming increasingly important in the management of inflammatory bowel disease (IBD). Ultrasonography is noninvasive, inexpensive, widely available, and does not require the use of ionizing radiation; as a result, it is particularly well accepted and tolerated by patients. Ultrasonography of IBD patients requires convex array probes of 3-5 MHz and higher frequency linear array probes from 5-15 MHz that allow adequate assessment of the five-layer wall pattern of the gastrointestinal tract [1]. Most parts of the large bowel, as well as major parts of the small bowel, can be easily visualized by IUS. The rectum and the proximal jejunum may sometimes be difficult to assess because of overlying structures. The advantages of ultrasonography include the rapid evaluation

Department of Gastroenterology, Lüneburg Hospital, University of Hamburg, Germany

\section{Conflict of Interest: None}

Correspondence to: Torsten Kucharzik, MD, PhD, Professor of Medicine, Department of Gastroenterology, Klinikum Lüneburg, University of Hamburg, Bögelstr. 1, 21339 Lüneburg, Germany, Tel.: +49 4131 772241, Fax: +49 4131 772245, e-mail: Torsten.Kucharzik@klinikum-lueneburg.de

Received 16 May 2016; accepted 18 October 2016; published online 15 November 2016

DOI: https://doi.org/10.20524/aog.2016.0105 of bowel wall thickness, as well as stratification that reflects alterations in histopathology in Crohn's disease (CD) and ulcerative colitis (UC). Visualization of the vascularization of the bowel using color Doppler sonography is the second advantage of IUS. The third major advantage, in comparison to other cross-sectional imaging modalities, includes the direct visualization of motility.

While in the past IUS has only been practiced by a few radiologists and by some specialists in ultrasound, it is now performed by gastroenterologists and by IBD specialists themselves in more and more countries. Educational programs on the use of IUS help promote this fascinating method. This review summarizes the different features of IUS and indications for its use in patients with CD and UC.

\section{Features of IUS in IBD}

A variety of parameters have been defined that characterize intestinal inflammation in IBD. The most prominent parameter during IUS for the detection of inflammatory activity within the intestine is bowel wall thickness, which mostly correlates well with clinical activity markers such as the Harvey Bradshaw index (HBI) and the CD activity index (CDAI) [2,3]. Common cutoff values are $2 \mathrm{~mm}$ of wall thickness for the small intestine and $3-4 \mathrm{~mm}$ for the large intestine. It has to be taken into account, however, that the bowel wall is usually slightly more prominent in the left colon than in the right colon. The sensitivity of bowel wall thickness as a parameter of inflammation could be increased if a lower 
cutoff value of $3 \mathrm{~mm}$ were to be used. Once the cutoff value is increased up to $4 \mathrm{~mm}$, sensitivity decreases from $88 \%$ to $75 \%$, whereas specificity increases from $93 \%$ to $97 \%$ [4]. Even though bowel wall thickness is the most common parameter for detecting intestinal inflammation, there is still a lack of standardization of measurement. Up to now, there is no international agreement as to where to perform the measurement and whether a transverse or a longitudinal section is required. It is therefore not surprising that the interobserver variability might differ between different examiners, depending on whether or not standardized parameters have been defined. Recent investigations have shown that interobserver variability may vary between high interobserver agreement $(\kappa=0.72-1)$ if measurement is standardized, and moderate agreement if no standards have been defined beforehand $(\kappa=0.54)[5,6]$. Other parameters of the bowel wall include the description of the overall echotexture and the different layers. An abrogation of the typical bowel wall stratification is usually an indicator of active inflammation. While the echotexture of the thickened bowel wall in patients with UC is mostly proportioned (Fig. 1), disproportioning of the thickened bowel wall is usually visible in CD. Even though abrogation of bowel stratification is a very common parameter in IUS, the interobserver agreement is very low in different studies because of the lack of standardization in findings $[5,6]$. The rigidity of the bowel wall can also be determined using B-mode ultrasonography. New techniques such as elastography might improve the evaluation of wall rigidity in IUS [7]. Strictures are usually defined by segmental thickening of the bowel wall. Defining a stenosis also requires the occurrence of prestenotic dilatation.

As intestinal inflammation in IBD is usually not restricted to the bowel wall, it is always useful to evaluate and to describe the surrounding structures, which have very characteristic features in IBD patients. Extramural changes within the mesenteric fat might give more information on the inflammatory status of a thickened bowel segment than do alterations of the bowel wall itself. The most prominent parameter in $\mathrm{CD}$ is the occurrence of fibrofatty proliferation, which is always a sign of active inflammation. It occurs rapidly during an acute flare of disease and disappears, at least in part, early after resolution of the intestinal inflammation. Fibrofatty proliferation appears as a white, hyperechoic zone surrounding the inflamed bowel (Fig. 2). Hypoechoic zones within the proliferated mesenteric fat are called mesenteric streaks and are indicators of severe inflammation in CD. Even though changes within the mesenteric fat can be detected easily, there are no standardized features to differentiate the severity of disease activity. This might be the reason why interobserver variability for this parameter is high when different studies are compared $[5,6]$. Transmural inflammation can also result in the occurrence of ascites. Mesenteric lymph nodes are another nonspecific sign of intestinal inflammation that may be seen during active as well as chronic intestinal inflammation, particularly in CD. Mesenteric lymphadenopathy usually remains visible even in quiescent disease and is therefore not a good parameter of activity for CD.

Another characteristic feature of intestinal inflammation and the thickened bowel wall is the increased vascularization, which may be visualized using color Doppler. Increased vascularization is always a sign of active inflammation that correlates with endoscopy, histology, and with the CDAI $[6,8,9]$. Quantification of vascularization has turned out to be difficult, as vascularization is always dependent on a variety of parameters, including feeding status. A semiquantitative characterization of vascularization may be made using the simple Limberg score, which divides visible vascularization during color Doppler into four different categories [10] (Fig. 3).

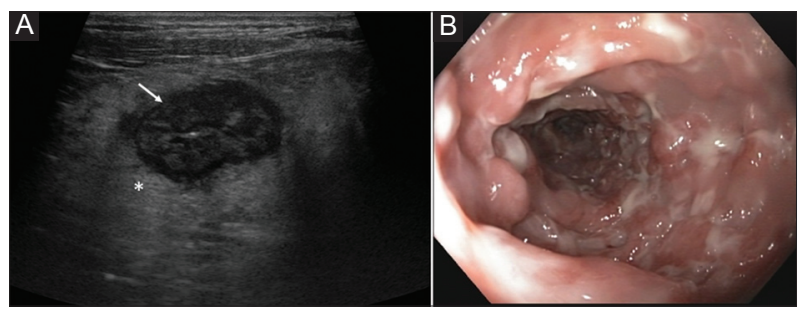

Figure 1 (A) Cross-sectional image of the sigma above the iliac artery (asterisk) in ulcerative colitis ( $9 \mathrm{MHz}$ probe) Hyperechoic thickening of the submucosa (arrow). (B) Corresponding endoscopic view of the same patient during the same week

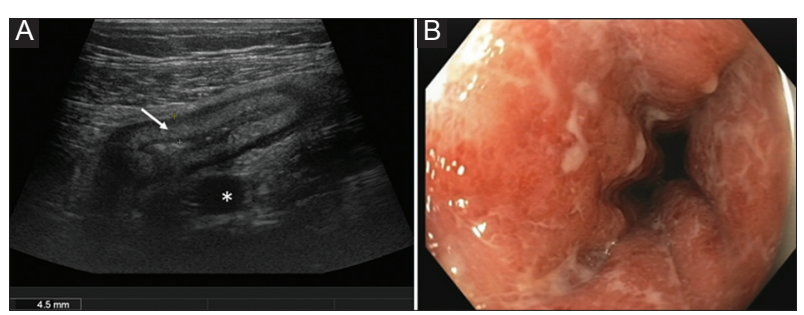

Figure 2 (A) Cross-sectional image of acute Crohn's colitis in the sigmoid colon ( $9 \mathrm{MHz}$ probe) Blurred stratification of the thickened bowel wall (arrow) and marked fibro-fatty proliferation (asterisk). (B) Corresponding endoscopic view of the same patient during the same week

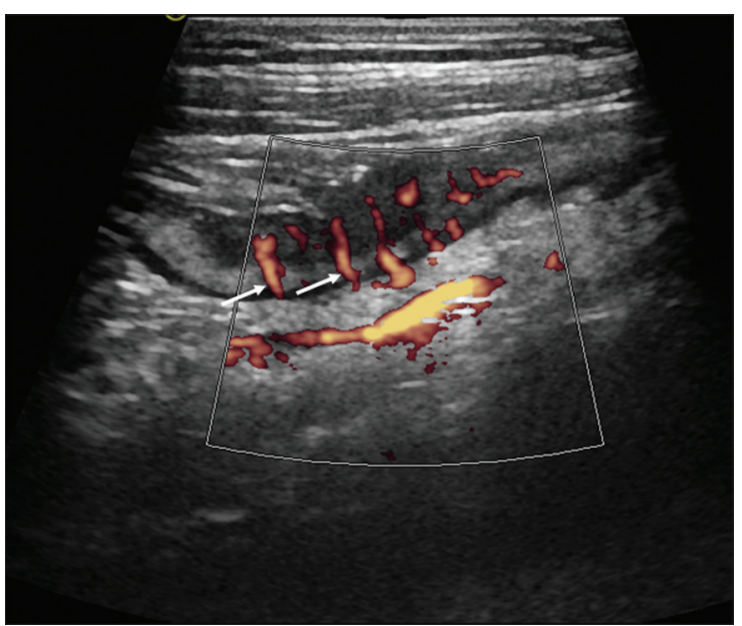

Figure 3 Longitudinal view of the terminal ileum in ileal Crohn's disease with Power Doppler (10 MHz probe) "Comb sign": parallel mesenteric vessels as a sign of severe inflammation (Limberg Score 4) (arrows) 


\section{Additional tools in bowel ultrasonography}

\section{Contrast-enhanced ultrasonography (CEUS)}

Another way to characterize vascularization in the bowel is by using CEUS, which allows a quantification of vascularization that directly correlates with inflammatory markers, such as C-reactive protein (CRP), or with the CDAI in CD patients [11-14]. CEUS has been shown to be a useful addition and a helpful tool for specific indications in IBD patients. In particular, CEUS has been shown to more precisely determine disease activity in CD patients [15-19]. The guidelines of the European Federation of Societies for Ultrasound in Medicine and Biology have recently specified particular indications for the use of CEUS in IBD patients, including the following: 1) estimation of disease activity in IBD; 2) distinguishing between fibrosis and inflammatory strictures in $\mathrm{CD}$; 3 ) characterization of suspected abscesses; and 4) confirming and following the route of a fistula [20].

CEUS has been shown to be capable of determining bowel wall vascularity in patients with $\mathrm{CD}$ and the findings correlate well with those of magnetic resonance imaging (MRI) [17,21,22]. The time-intensity curve analysis, involving the determination of peak intensity, area under the curve and time to peak intensity using specific software, may help quantify disease activity. Quantitative measurement of bowel wall vascularization, determined by CEUS, correlates well with endoscopic severity [23] and may predict disease activity in IBD [18]. During biological therapy, changes in bowel wall enhancement may be a useful modality for monitoring disease activity in CD [23]. The most useful indication for CEUS in the daily management of CD patients is probably the differentiation of abscesses from inflammatory infiltrates. CEUS allows a rapid and precise differentiation of abscesses from phlegmons [24]. An additional advantage of CEUS is that any abscesses detected can immediately be targeted for diagnostic, or even therapeutic puncture or drainage within the same examination.

Injection of contrast medium into the orifice of a fistula may improve the visualization of the route of the fistula tract in $\mathrm{CD}$ [25]. An easier method for visualizing the fistula tract is the injection of carbonated water into the orifice of the fistula. A limitation of CEUS in IUS is that intestinal motility might impair image quality and that only specific bowel segments can be evaluated during CEUS. CEUS is still not broadly used during IUS in IBD patients and the current use of CEUS is limited to specific clinical situations.

\section{Elastography}

Ultrasound elasticity imaging is based on strain and the deformation and elastic moduli that have been clinically developed and approved for the evaluation of different tissues. Different methods have been devised to determine elasticity using ultrasound. In one, shear waves are generated by an ultrasound transducer that produces an acoustic radiation force impulse (ARFI). ARFI elastography has been used to assess a variety of human tissues and has been shown to discriminate low-grade fibrosis from high-grade fibrosis in exvivo human intestinal specimens, based on their biochemical elastic properties [26]. Another method performs real-time elastography of the intestine using fast reconstruction of tissue elastic moduli [7].

Differentiation between fibrotic and inflammatory stenosis is a difficult issue in patients with fibrostenotic CD. Today, there is no gold standard method to differentiate between inflammatory and fibrostenotic strictures. In a recent study, ultrasound elastography-derived shear wave velocity (SWV) was helpful in distinguishing acute inflamed from fibrotic bowel in a 2,4,5-trinitrobenzenesulfonic acid-induced rat model [27]. In this trial, the mean bowel wall SWV was significantly higher for fibrotic than for acute inflammatory changes in the gut. The area under the receiver operating characteristic (ROC) curve of the SWV ratio for differentiating histopathologically confirmed fibrotic from inflamed bowel was 0.971 .

In a recent study, ultrasound-based real-time elastography (RTE) was compared pre-, intra- and postoperatively with surgical resections, histologically and by measurement of the collagen content. It was shown that RTE can be used to reliably distinguish fibrotic from non-fibrotic tissue $(\mathrm{P}<0.001)$ [7]. There was a good correlation between pre-, intra- and postoperative RTE strain measurements of unaffected and affected bowel segments in this trial.

Further studies with this interesting method have to be conducted to confirm these results before it can be used for clinically based decision-making in patients with stenotic $\mathrm{CD}$. Whether it will ever be clearly possible to discriminate inflammatory from fibrostenotic stenosis in CD in the future remains questionable, as stenosis in $\mathrm{CD}$ always involves a combination of inflammatory and fibrotic tissue activity [28].

\section{When to use IUS in IBD patients}

\section{Primary diagnosis of $C D$}

IUS has been demonstrated to have high sensitivity and specificity in the diagnosis of $\mathrm{CD}$ and in the detection of its complications, including stenoses, as well as of fistulas or abscess formations [29-37]. The most prominent and most important parameter that indicates inflammatory activity in $\mathrm{CD}$ is an increase in bowel wall thickness [38]. High sensitivity in determining an increase in bowel wall thickness in various parts of the intestine has been shown in several studies, using endoscopy as comparator, and varies between $75-93 \%$, with specificities of $75-100 \%[39,40]$. A recent study in 249 patients with suspected $\mathrm{CD}$, using ileocolonoscopy and MRI as comparators, was able to demonstrate a high diagnostic value for IUS in the detection of small bowel CD, with a sensitivity of $94 \%$, a specificity of $97 \%$, a positive predictive value of $97 \%$, and a negative predictive value of $94 \%$ [41]. The overall sensitivity of IUS in the diagnosis of CD has been analyzed in a meta-analysis and assessed using a summary ROC curve; the area under the curve was 0.94 , indicating good diagnostic accuracy [32]. In 
the most recent meta-analysis, IUS showed $79.7 \%$ sensitivity and $96.7 \%$ specificity in the diagnosis of suspected CD, and $89 \%$ sensitivity and $94.3 \%$ specificity in the initial assessment of patients with established CD. In this study, IUS identified ileal CD with $92.7 \%$ sensitivity and $88.2 \%$ specificity, and colonic CD with $81.8 \%$ sensitivity and $95.3 \%$ specificity, having a lower accuracy in the detection of proximal lesions [37]. These studies indicate that IUS is a valuable method for detecting CD in the primary evaluation of patients with intestinal symptoms suggestive of $\mathrm{CD}$. It has also been shown that its high negative predictive value can exclude $\mathrm{CD}$ in patients with non-specific gastrointestinal symptoms and normal biomarkers, such as CRP and calprotectin. A recent study provided evidence that ultrasonography in combination with ileocolonoscopy is the most accurate and most cost-effective diagnostic procedure, in comparison with MRI, for the evaluation of patients with suspected CD [42].

Currently, the gold standard for IBD diagnosis is not clearly defined. Previous studies compared IUS to other single comparators, such as endoscopy or MRI. However, we have to keep in mind that these comparators can only serve as surrogate markers. The diagnosis of IBD is confirmed by clinical evaluation and by a combination of other investigative modalities, including history, biomarkers, endoscopy, and cross-sectional imaging such as IUS. As a current methodology with a high negative predictive value, IUS is accepted as a first-line tool in the primary diagnosis workup of adults and children with suspected small bowel inflammation [30,43-46]. If the appropriate expertise is available, IUS could also be used as a primary diagnostic modality in adult CD patients and MRI could be reserved for patients with unclear findings or symptoms.

The sensitivity of ultrasonography during primary diagnosis can even be increased by using CEUS, as was demonstrated in recent studies showing that detection of increased bowel wall thickness in CD patients had $~ 90 \%$ sensitivity and $100 \%$ and $94 \%$ specificity, respectively [19]. The value of CEUS during IUS is further outlined below.

\section{Detection of CD extent and localization}

Several studies have investigated the value of IUS in determining the location of $\mathrm{CD}$ within the small and large bowel, as well as the extent of the disease. Data from 8 trials using endoscopy as reference standard showed a sensitivity for IUS in the range $74-96 \%$ with a specificity of $67-98 \%$. The pooled sensitivity from these data was $86 \%$ (95\% CI $83-88 \%$ ) with a pooled specificity of $94 \%$ (95\% CI $93-95 \%)$, demonstrating the high value of IUS for determining the extent of CD [47].

Weaknesses of IUS include the detection of CD in different parts of the intestine, including the proximal jejunum and the rectum [48]. Detection of small bowel lesions in CD might be improved by small intestinal CEUS (SICUS). A recent study demonstrated that, particularly within the jejunum, the detection rate of IUS could be markedly improved from
$80 \%$ to $100 \%$ by using SICUS with $500-700 \mathrm{~mL}$ polyethylene glycol solution [49]. These findings have been confirmed by others [50].

\section{Assessment of complications in CD}

Extramural complications in $\mathrm{CD}$, such as abscesses and fistulas, can easily be detected during IUS. Abscesses usually appear as an irregular, aperistaltic, hypoechoic zone without vascularization and only a few internal echoes (Fig. 4). Abscesses sometimes contain air that appears as hyperechoic streaks. Fistulas are hypoechoic tracts between bowel loops, or at least with the origin in the bowel, and are sometimes connected to other tissue or organs such as the skin, the cystic bladder, or the vagina. They may contain air as a small hyperechoic zone (Fig. 5). The sensitivity to detect abscesses in $\mathrm{CD}$ has been determined in a recent meta-analysis summarizing data from three studies that used surgery as comparator [47]. The sensitivity varied between $80-100 \%$ and the specificity between $92-94 \%[33,35,51]$. The pooled sensitivity was $84 \%$ (95\% CI $79-88 \%)$ and the pooled specificity was 93\% (95\% CI 89-95\%) [47].

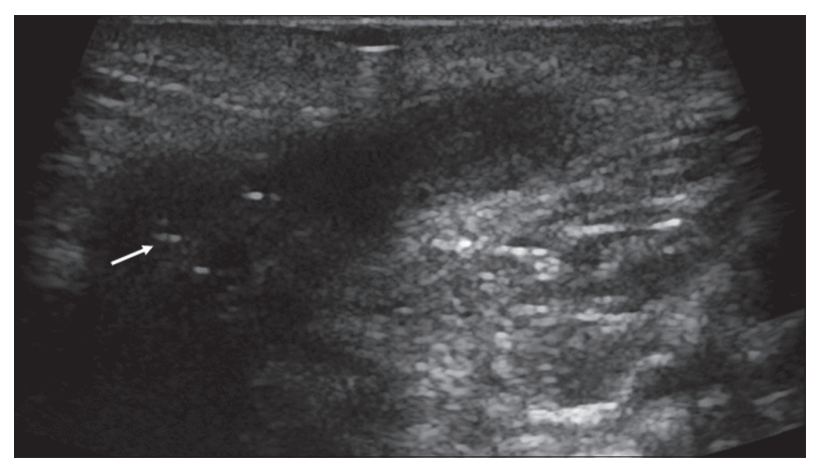

Figure 4 Perianal positioning of a $10 \mathrm{MHz}$ probe. Perianal abscess with hyperechoic streaks (arrow) in Crohn's disease

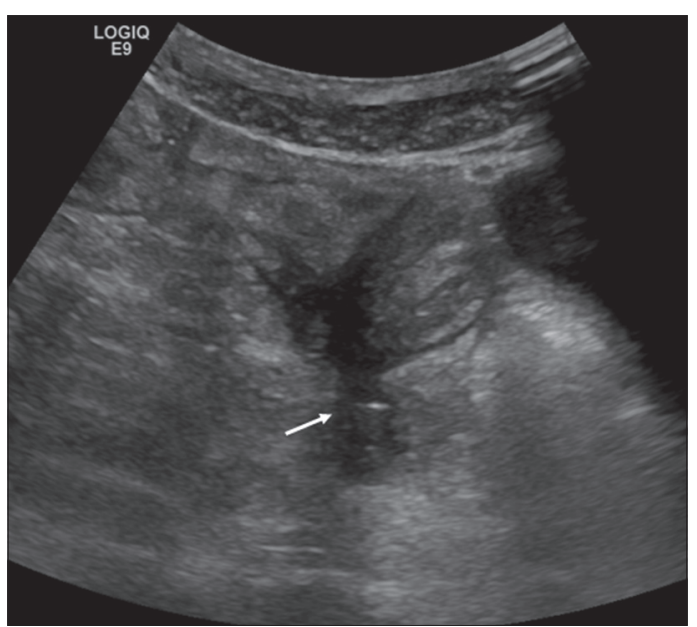

Figure 5 Fistula (arrow) in ileal Crohn's disease ( $9 \mathrm{MHz}$ probe) 
The detection rate of fistulas depends on their localization, with a sensitivity between $67 \%$ and $82 \%$ and a specificity between $90 \%$ and $100 \%$, depending on different trials [33-36,51]. A recent meta-analysis that summarized the results of four different ultrasound trials for diagnosing fistulas revealed a pooled sensitivity of $74 \%$ (95\% CI 67-79\%) and a specificity of $95 \%$ (95\% CI 91-97\%) [47]. As outlined below, the identification and characterization of suspected abscesses appears to be a good indication for the use of CEUS [20]. A recent retrospective analysis in 50 patients determined a specificity for the detection of abscesses of $100 \%$, with a correlation coefficient of 0.974 between CEUS and other techniques [24].

IUS has also been shown to have a high sensitivity and specificity in the diagnosis of stenosis affecting the large and the small bowel [30]. The sensitivity in three studies that used surgery as reference varied between $75-100 \%$, with a specificity between $89-91 \%$, a pooled sensitivity of $79 \%$ (95\% CI 71-84\%) and a pooled specificity of $92 \%$ (95\% CI 87-96\%) [33-36,52]. The sensitivity for detecting stenoses in CD may be increased from $74 \%$ to $89 \%$ by using SICUS [53]. However, performing SICUS is not a standard method during the examination of the small bowel and is usually only required for a few indications.

The differentiation between predominately inflammatory versus fibrotic strictures is a frequent clinical challenge in patients with CD. It has recently been suggested that CEUS might be able to differentiate between these two categories [30]. Recent data also suggest that CEUS may help distinguish between inflammatory stenosis and more fibrotic stenosis, where contrast enhancement is lower than in the more inflammatory structures [54]. However, existing data are controversial, and other investigators did not report any additional benefit during the quantitative assessment of stenotic bowel wall segments using CEUS [55]. As stenosis is always a mixture of inflammatory and fibrotic components, it remains questionable whether useful differentiation can be achieved with any of the current diagnostic modalities $[28,56]$. Table 1 summarizes data on the detection of mural and extramural complications in CD with IUS.

The excellent data from various studies appear to justify the view that, in centers with appropriate expertise, IUS might be used as a primary diagnostic imaging modality in CD patients to detect or to exclude mural and extramural complications. MRI or CT might be restricted to unclear clinical situations.

\section{Monitoring disease activity in CD}

Recent trials have proven that IUS has a role in monitoring the disease scores of CD [32]. It has been shown that IUS has the ability to monitor the response to biological treatment [57]. IUS may also predict an outcome independently of other disease activity markers in UC $[58,59]$. However, it has to be noted that there is no gold standard for IBD activity, and that any types of diagnostic modality, including endoscopy, MRI, laboratory parameters or IUS, could only be used as surrogate markers in this situation. There are only a few relatively small studies that have evaluated the usefulness of IUS in monitoring therapeutic responses in CD. A study with 21 patients used ultrasonography to assess bowel wall thickness and vascularization after treatment with anti-tumor necrosis factor (TNF) [60]. In this trial, antiTNF therapy was associated with a significant reduction in bowel wall thickness, as well as in vascularization determined by Doppler flow. Ultrasound parameters showed improvement in $50 \%$ of the patients in this trial. Another small study used SICUS to show that, during anti-inflammatory treatment, changes on IUS correlated significantly with the CDAI [57]. A more recent prospective trial evaluated ultrasonographic features in patients with $\mathrm{CD}$ after treatment with biologicals, using ileocolonoscopy as reference standard [61]. In this trial, normalization of the IUS parameters could be observed in $62.8 \%$ of the patients, with a significant correlation compared to ileocolonoscopy $(\kappa=0.76, \mathrm{P}<0.001)$.

Some authors suggest that CEUS might be useful for evaluating treatment outcomes shortly after initiating treatment with biologicals [14]. A large multicenter trial, including 243 patients from 50 centers in Germany, has recently been conducted to determine the role of IUS in monitoring treatment response [62]. In this trial, CD patients with an acute flare of disease received anti-inflammatory treatment; most of them had been treated with anti-TNF therapy with a significant drop in HBI after three months. Almost all sonographic parameters determined during IUS, including bowel wall thickness, vascularization parameters, fibro-fatty proliferation and other parameters, showed a highly significant decrease $(\mathrm{P}<0.001$ in all groups) at different sites. Improvement in ultrasound parameters correlated with laboratory parameters such as CRP [62]. These data could prove that ultrasonography is indeed a very useful method for use in the follow up of patients with CD and the determination of early therapeutic responses (Fig. 6).

Based on current studies, IUS seems to be a valuable method for evaluating transmural healing in CD [63]. A recent investigation evaluated quantitative parameters to determine the degree of bowel damage during chronic intestinal inflammation and transmural healing in CD [64]. However, it has to be noted that the profound alterations of transmural inflammation during chronic CD rarely completely normalize, even after treatment with immunosuppressive or biological agents [62]. In a recent study involving 66 patients, transmural healing as determined by IUS could only be detected in $25 \%$ of patients receiving biologicals, while mucosal healing was detected in $38 \%$. Nevertheless, the correlation was statistically significant [65]. Based on the recent studies, IUS is increasingly becoming the method of choice in the follow up of CD patients to monitor transmural healing in response to treatment.

\section{Evaluation of postoperative disease recurrence}

For decades, endoscopy has been the gold standard in the determination of postoperative recurrence of $\mathrm{CD}$. The Rutgeerts score has been proved to be a valid endoscopic score of activity for postoperative recurrence [66]. More recently, ultrasonography has also proved capable of detecting postoperative recurrence. A good correlation with the Rutgeerts score could be determined using SICUS, 
Table 1 Intestinal ultrasound and detection of mural and extramural complications in Crohn's disease

\begin{tabular}{|c|c|c|c|c|c|c|}
\hline Complication & Author & $\begin{array}{c}\text { Number } \\
\text { of } \\
\text { patients }\end{array}$ & $\begin{array}{l}\text { Number of } \\
\text { complications }\end{array}$ & Comparator & Sensitivity (\%) & Specificity (\%) \\
\hline \multicolumn{7}{|l|}{ Stenosis } \\
\hline & Gasche et al 1999 [33] & 33 & 22 & Surgery & 100 & 90 \\
\hline & Maconi et al 1996 [34] & 98 & 40 & Surgery, colonoscopy, CT & 75 & 93 \\
\hline & Kohn et al 1999 [35] & 25 & 16 & Surgery & 75 & 89 \\
\hline & Parente et al 2002 [53] & 296 & 75 & Surgery, X-ray studies & 79 & 98 \\
\hline & $\begin{array}{l}\text { Calabrese et al } \\
2013[36]\end{array}$ & 59 & & CT-enteroclysis & 96 & 80 \\
\hline \multicolumn{7}{|l|}{ Abscess } \\
\hline & Gasche et al 1999 [33] & 33 & 9 & Surgery & 100 & 92 \\
\hline & Maconi et al 2003 [51] & 128 & 26 & Surgery & 91 & 93 \\
\hline & Maconi et al 1996 [34] & 98 & 6 & Surgery, colonoscopy, CT & 83 & 94 \\
\hline & $\begin{array}{l}\text { Calabrese et al } \\
2013[36]\end{array}$ & 59 & & CT-enteroclysis & 78 & 100 \\
\hline \multicolumn{7}{|l|}{ Fistula } \\
\hline & $\begin{array}{l}\text { Martinez et al } \\
2009[87]\end{array}$ & 30 & 17 & $\begin{array}{l}\text { Surgery, barium studies, } \\
\text { colonoscopy }\end{array}$ & 82 & 100 \\
\hline & Gasche et al 1999 [33] & 33 & 23 & Surgery & 87 & 90 \\
\hline & Maconi et al 2003 [51] & 128 & 56 & Surgery & 71 & 96 \\
\hline & Kohn et al 1999 [35] & 25 & 3 & Surgery & 67 & 95 \\
\hline & $\begin{array}{l}\text { Calabrese et al } \\
2013[36]\end{array}$ & 59 & & CT-enteroclysis & 79 & 96 \\
\hline
\end{tabular}
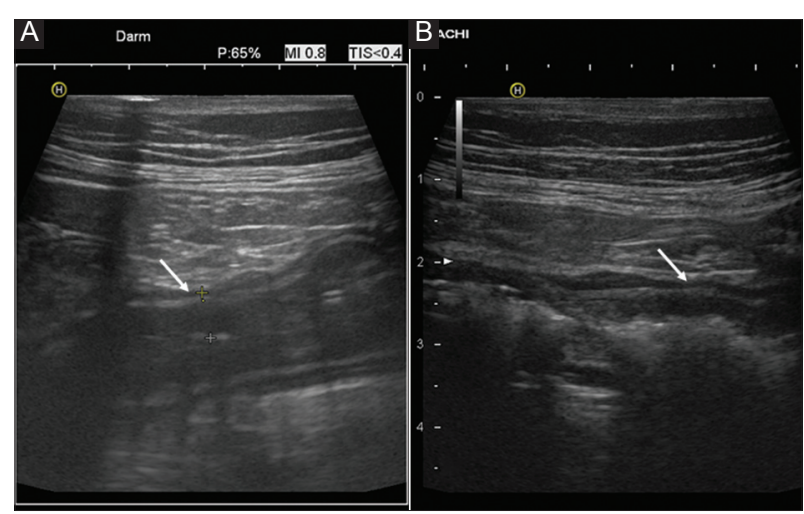

Figure 6 Changes in ultrasound findings after anti-inflammatory treatment ( $9 \mathrm{MHz}$ probe) Joining pretherapeutic image (A) into actual finding (B) at the same site after 2 weeks of treatment with anti-TNF in acute Crohn's disease: reduction of bowel wall thickness and mucosal swelling (arrows)

with a positive predictive value of $87 \%$ for the detection of postoperative disease recurrence and a good correlation with the Rutgeerts score $(\mathrm{r}=0.67, \mathrm{P}=0.0001)$ [2]. A more recent study showed that the sensitivity in detecting postoperative recurrence using conventional IUS was $89.7 \%$, which could be increased up to $98 \%$ by using CEUS [60]. Detailed information on the anastomosis that has been performed is mandatory for interpreting the sonographic findings in a useful manner.

In the recent POCER trial, for excluding relevant intestinal inflammation postoperatively, calprotectin has also been shown to correlate well with endoscopic disease activity in a postoperative setting, having a high negative predictive value of up to $91 \%$ [67]. In the future, a combination of IUS plus calprotectin might be a valuable parameter for determining postoperative recurrence in $\mathrm{CD}$ patients. This has to be further evaluated in prospective trials.

\section{Prognostic value of IUS in CD}

Various authors have tried to identify the prognostic value of ultrasonography for the recurrence of $\mathrm{CD}$ and to develop prognostic markers and features of IUS in IBD. It has been demonstrated that IUS has the capacity to determine the risk of recurrence in a highly specific and sensitive manner [3,68-71]. The prognostic value can even be enhanced by performing SICUS with the use of oral contrast, which allows better distension of the small and large bowel and might improve the prognostic value of ultrasonography [72-74].

Most recently, the use of CEUS has been shown to be a valuable method for predicting the treatment outcome in 
patients with $\mathrm{CD}$, by measuring bowel perfusion with dynamic CEUS [14].

\section{Rectal involvement in CD}

\section{Perianal ultrasonography and transrectal ultrasonography (TRUS)}

Perianal fistulizing disease occurs frequently in patients with $\mathrm{CD}$, with a prevalence ranging from $10 \%$ at the time of diagnosis up to $20 \%$ after 20 years of the disease course $[75,76]$. The diagnosis of perianal disease and its classification are often made using a combination of clinical and imaging findings. Apart from examination under anesthesia as a previously described gold standard, MRI and TRUS are currently used as methods for the detection and classification of perianal CD. Recent studies have shown that the accuracy of TRUS is comparable with that of MRI in perianal fistulizing disease ( $87 \%$ for MRI and $91 \%$ for TRUS) [77]. These data were confirmed in a meta-analysis that found no relevant difference between the sensitivities of MRI and TRUS (87\%, 95\% CI 0.63-0.96 vs. $87 \%$, 95\% CI $0.70-0.95)$, whereas the specificity for MRI and TRUS differed slightly (69\% for MRI and $43 \%$ for TRUS) [78]. The disadvantage of TRUS is the fact that it is invasive and uncomfortable. Although MRI of the pelvis is an established modality, timely access and costs remain problematic. As both methods require expensive equipment and experienced investigators, perianal ultrasonography has been suggested as an alternative method with high sensitivity and specificity in perianal $C D$. In a recent study investigating 46 patients with perianal CD, using TRUS as gold standard, 53 fistula detected by TRUS were correctly classified by perianal ultrasound in 45 cases, reaching a sensitivity of $84.9 \%$. Perianal ultrasound showed 10 perianal abscesses, which could all be confirmed by TRUS, and TRUS found no further abscesses [79]. The sensitivity of perianal ultrasonography in perianal CD with or without contrast medium has also been shown by other investigators [80-83]. Perianal ultrasound therefore appears to be a simple and painless method for detecting and classifying perianal and rectovaginal fistulas or abscesses in real time in patients with perianal CD (Fig. 4) [79].

\section{Ultrasonography in UC}

The value of ultrasonography in the primary diagnosis and follow up of UC, compared to $\mathrm{CD}$, has been much less evaluated. Part of the reason may be that, particularly during mild left-sided UC, the bowel wall is sometimes not thickened and the inflammation is only restricted to the mucosa. The most characteristic feature of inflammation in UC is thickening of the bowel wall, as mentioned earlier. In contrast to $\mathrm{CD}$, the thickening of the bowel wall is mostly proportional and stratification of the bowel wall is usually preserved.
While it is not surprising that thickening of the mucosa is a characteristic sign of inflammation, it is also useful to visualize thickening of the submucosa (Fig. 2). Hyperechoic thickening of the submucosa reflects edema during active inflammation in UC. Although it is known from clinical practice that thickening of the bowel wall can be determined as an early sign of inflammation in UC that disappears after resolution of the intestinal inflammation, there is a surprising shortage of data in the literature describing the course of bowel wall thickness after treatment, and in particular the time course of improvement. The exact value of IUS in the follow up of patients with active UC is currently being investigated in a multicenter trial in Germany, including 50 different IBD centers.

Initial data suggest that Doppler ultrasonography of the superior and inferior mesenteric arteries could be used to evaluate disease activity in UC, as well as to determine the risk of relapse [84-86]. However, this technique has only been used in very few centers up to now and the results have not yet been confirmed by other investigators.

Other ultrasonographic features in IBD that are useful in $\mathrm{CD}$, including the determination of vascularization and the evaluation of surrounding structures such as fibrofatty proliferation, seem to be less useful in UC. However, during severely active UC, extramural signs of inflammation, such as fibrofatty proliferation, ascites or even mesenteric streaks, might be determined in specific situations, even though UC is usually restricted to the mucosal site.

\section{Concluding remarks}

During the last couple of years, IUS has become a useful and well defined method that is helpful in the management of IBD patients. The improved quality of ultrasound equipment, with better image quality and high-resolution probes for an acceptable budget, has expanded the use of IUS among gastroenterologists during recent years. IUS in IBD now has widely accepted indications and is part of national and European guidelines. Indications include the primary diagnosis of CD and UC, as well as the detection of extramural complications, detection and evaluation of stenotic strictures, and follow up of the disease course. Additional tools, such as CEUS, elastography or perianal ultrasound might be helpful in various clinical situations.

\section{References}

1. StrobelD, Goertz RS, Bernatik T. Diagnostics in inflammatory bowel disease: ultrasound. World J Gastroenterol 2011;17:3192-3197.

2. Calabrese E, Petruzziello C, Onali S, et al. Severity of postoperative recurrence in Crohn's disease: correlation between endoscopic and sonographic findings. Inflamm Bowel Dis 2009;15:1635-1642.

3. Rigazio C, Ercole E, Laudi C, et al. Abdominal bowel ultrasound can predict the risk of surgery in Crohn's disease: proposal of an ultrasonographic score. Scand J Gastroenterol 2009;44:585-593.

4. Fraquelli M, Colli A, Casazza G, et al. Role of US in detection of 
Crohn disease: meta-analysis. Radiology 2005;236:95-101.

5. Calabrese E, Kucharzik T, Maaser C, et al. Inter-observer agreement in bowel ultrasonography for diagnostic assessment in patients with Crohn's disease. Gastroenterology 2016;150(Suppl 1):S433-S434.

6. Fraquelli M, Sarno A, Girelli C, et al. Reproducibility of bowel ultrasonography in the evaluation of Crohn's disease. Dig Liver Dis 2008;40:860-866.

7. Baumgart DC, Müller HP, Grittner U, et al. US-based real-time elastography for the detection of fibrotic gut tissue in patients with stricturing Crohn disease. Radiology 2015;275:889-899.

8. Drews $\mathrm{BH}$, Barth TF, Hänle $\mathrm{MM}$, et al. Comparison of sonographically measured bowel wall vascularity, histology, and disease activity in Crohn's disease. Eur Radiol 2009;19:1379-1386.

9. Neye H, Voderholzer W, Rickes S, Weber J, Wermke W, Lochs H. Evaluation of criteria for the activity of Crohn's disease by power Doppler sonography. Dig Dis 2004;22:67-72.

10. Limberg B, Osswald B. Diagnosis and differential diagnosis of ulcerative colitis and Crohn's disease by hydrocolonic sonography. Am J Gastroenterol 1994;89:1051-1057.

11. Migaleddu V, Quaia E, Scanu D, et al. Inflammatory activity in Crohn's disease: CE-US. Abdom Imaging 2011;36:142-148.

12. Medellin-Kowalewski A, Wilkens R, Wilson A, Ruan J, Wilson SR. Quantitative contrast-enhanced ultrasound parameters in Crohn disease: their role in disease activity determination with ultrasound. AJR Am J Roentgenol 2016;206:64-73.

13. Ma X, Li Y, Jia H, et al. Contrast-enhanced ultrasound in the diagnosis of patients suspected of having active Crohn's disease: meta-analysis. Ultrasound Med Biol 2015;41:659-668.

14. Saevik F, Nylund K, Hausken T, Ødegaard S, Gilja OH. Bowel perfusion measured with dynamic contrast-enhanced ultrasound predicts treatment outcome in patients with Crohn's disease. Inflamm Bowel Dis 2014;20:2029-2037.

15. Kratzer W, Schmidt SA, Mittrach C, et al. Contrast-enhanced wideband harmonic imaging ultrasound (SonoVue): a new technique for quantifying bowel wall vascularity in Crohn's disease. Scand J Gastroenterol 2005;40:985-991.

16. Robotti D, Cammarota T, Debani P, Sarno A, Astegiano M. Activity of Crohn disease: value of Color-Power-Doppler and contrastenhanced ultrasonography. Abdom Imaging 2004;29:648-652.

17. Schlottmann K, Kratzer W, Schölmerich J. Doppler ultrasound and intravenous contrast agents in gastrointestinal tract disorders: current role and future implications. Eur J Gastroenterol Hepatol 2005;17:263-275.

18. Romanini L, Passamonti $M$, Navarria $M$, et al. Quantitative analysis of contrast-enhanced ultrasonography of the bowel wall can predict disease activity in inflammatory bowel disease. Eur $J$ Radiol 2014;83:1317-1323.

19. Migaleddu V, Scanu AM, Quaia E, et al. Contrast-enhanced ultrasonographic evaluation of inflammatory activity in Crohn's disease. Gastroenterology 2009;137:43-52.

20. Piscaglia F, Nolsøe C, Dietrich CF, et al. The EFSUMB Guidelines and Recommendations on the Clinical Practice of Contrast Enhanced Ultrasound (CEUS): update 2011 on non-hepatic applications. Ultraschall Med 2012;33:33-59.

21. Pauls S, Gabelmann A, Schmidt SA, et al. Evaluating bowel wall vascularity in Crohn's disease: a comparison of dynamic MRI and wideband harmonic imaging contrast-enhanced low MI ultrasound. Eur Radiol 2006;16:2410-2417.

22. Schreyer AG, Finkenzeller T, Gössmann H, et al. Microcirculation and perfusion with contrast enhanced ultrasound (CEUS) in Crohn's disease: first results with linear contrast harmonic imaging (CHI). Clin Hemorheol Microcirc 2008;40:143-155.

23. Ripollés T, Martínez MJ, Paredes JM, Blanc E, Flors L, Delgado F. Crohn disease: correlation of findings at contrast-enhanced US with severity at endoscopy. Radiology 2009;253:241-248.

24. Ripollés T, Martínez-Pérez MJ, Paredes JM, Vizuete J,
García-Martínez E, Jiménez-Restrepo DH. Contrast-enhanced ultrasound in the differentiation between phlegmon and abscess in Crohn's disease and other abdominal conditions. Eur J Radiol 2013;82:e525-e531.

25. Henrich W, Meckies J, Friedmann W. Demonstration of a rectovaginal fistula with the ultrasound contrast medium Echovist. Ultrasound Obstet Gynecol 2000;15:148-149.

26. Dillman JR, Stidham RW, Higgins PD, et al. Ultrasound shear wave elastography helps discriminate low-grade from high-grade bowel wall fibrosis in ex vivo human intestinal specimens. J Ultrasound Med 2014;33:2115-2123.

27. Dillman JR, Stidham RW, Higgins PD, Moons DS, Johnson LA, Rubin JM. US elastography-derived shear wave velocity helps distinguish acutely inflamed from fibrotic bowel in a Crohn disease animal model. Radiology 2013;267:757-766.

28. Rieder F, Zimmermann EM, Remzi FH, Sandborn WJ. Crohn's disease complicated by strictures: a systematic review. Gut 2013;62:1072-1084.

29. Hirche TO, Russler J, Schröder O, et al. The value of routinely performed ultrasonography in patients with Crohn disease. Scand J Gastroenterol 2002;37:1178-1183.

30. Panes J, Bouhnik Y, Reinisch W, et al. Imaging techniques for assessment of inflammatory bowel disease: joint ECCO and ESGAR evidence-based consensus guidelines. J Crohns Colitis 2013;7:556-585.

31. Horsthuis K, Bipat S, Bennink RJ, Stoker J. Inflammatory bowel disease diagnosed with US, MR, scintigraphy, and CT: metaanalysis of prospective studies. Radiology 2008;247:64-79.

32. Dong J, Wang $\mathrm{H}$, Zhao J, et al. Ultrasound as a diagnostic tool in detecting active Crohn's disease: a meta-analysis of prospective studies. Eur Radiol 2014;24:26-33.

33. Gasche C, Moser G, Turetschek K, Schober E, Moeschl P, Oberhuber G. Transabdominal bowel sonography for the detection of intestinal complications in Crohn's disease. Gut 1999;44:112-117.

34. Maconi G, Bollani S, Bianchi Porro G. Ultrasonographic detection of intestinal complications in Crohn's disease. Dig Dis Sci 1996;41:1643-1648.

35. Kohn A, Cerro P, Milite G, De Angelis E, Prantera C. Prospective evaluation of transabdominal bowel sonography in the diagnosis of intestinal obstruction in Crohn's disease: comparison with plain abdominal film and small bowel enteroclysis. Inflamm Bowel Dis 1999;5:153-157.

36. Calabrese E, Zorzi F, Onali S, et al. Accuracy of small-intestine contrast ultrasonography, compared with computed tomography enteroclysis, in characterizing lesions in patients with Crohn's disease. Clin Gastroenterol Hepatol 2013;11:950-955.

37. Calabrese E, Maaser C, Zorzi F, et al. Bowel ultrasonography in the management of Crohn's disease. A review with recommendations of an international panel of experts. Inflamm Bowel Dis 2016;22:1168-1183.

38. Di Mizio R, Maconi G, Romano S, D’Amario F, Bianchi Porro G, Grassi R. Small bowel Crohn disease: sonographic features. Abdom Imaging 2004;29:23-35.

39. Maconi G, Radice E, Greco S, Bianchi Porro G. Bowel ultrasound in Crohn's disease. Best Pract Res Clin Gastroenterol 2006;20:93-112.

40. Parente F, Greco S, Molteni M, Anderloni A, Maconi G, Bianchi Porro G. Modern imaging of Crohn's disease using bowel ultrasound. Inflamm Bowel Dis 2004;10:452-461.

41. Castiglione F, Mainenti PP, De Palma GD, et al. Noninvasive diagnosis of small bowel Crohn's disease: direct comparison of bowel sonography and magnetic resonance enterography. Inflamm Bowel Dis 2013;19:991-998.

42. Maconi G, Bolzoni E, Giussani A, Friedman AB, Duca P. Accuracy and cost of diagnostic strategies for patients with suspected Crohn's disease. J Crohns Colitis 2014;8:1684-1692.

43. Allgayer H, Braden B, Dietrich CF. Transabdominal ultrasound in 
inflammatory bowel disease. Conventional and recently developed techniques-update. Med Ultrason 2011;13:302-313.

44. Nylund K, Hausken T, Gilja OH. Ultrasound and inflammatory bowel disease. Ultrasound Q 2010;26:3-15.

45. Schreiber-Dietrich D, Chiorean L, Cui XW, et al. Particularities of Crohn's disease in pediatric patients: current status and perspectives regarding imaging modalities. Expert Rev Gastroenterol Hepatol 2015;9:1313-1325.

46. Kucharzik T, Petersen F, Maaser C. Bowel ultrasonography in inflammatory bowel disease. Dig Dis 2015;33 Suppl 1:17-25.

47. Panés J, Bouzas R, Chaparro M, et al. Systematic review: the use of ultrasonography, computed tomography and magnetic resonance imaging for the diagnosis, assessment of activity and abdominal complications of Crohn's disease. Aliment Pharmacol Ther 2011;34:125-145.

48. Parente F, Greco S, Molteni M, Anderloni A, Bianchi Porro G. Imaging inflammatory bowel disease using bowel ultrasound. Eur J Gastroenterol Hepatol 2005; 17:283-291.

49. Parente F, Greco S, Molteni M, et al. Oral contrast enhanced bowel ultrasonography in the assessment of small intestine Crohn's disease. A prospective comparison with conventional ultrasound, x ray studies, and ileocolonoscopy. Gut 2004;53:1652-1657.

50. Pallotta N, Tomei E, Viscido A, et al. Small intestine contrast ultrasonography: an alternative to radiology in the assessment of small bowel disease. Inflamm Bowel Dis 2005;11:146-153.

51. Maconi G, Sampietro GM, Parente F, et al. Contrast radiology, computed tomography and ultrasonography in detecting internal fistulas and intra-abdominal abscesses in Crohn's disease: a prospective comparative study. Am J Gastroenterol 2003;98:1545-1555.

52. Maconi G, Parente F, Bollani S, Cesana B, Bianchi Porro G. Abdominal ultrasound in the assessment of extent and activity of Crohn's disease: clinical significance and implication of bowel wall thickening. Am J Gastroenterol 1996;91:1604-1609.

53. Parente F, Maconi G, Bollani S, et al. Bowel ultrasound in assessment of Crohn's disease and detection of related small bowel strictures: a prospective comparative study versus $\mathrm{x}$ ray and intraoperative findings. Gut 2002;50:490-495.

54. Wilson SR, Burns PN. Microbubble-enhanced US in body imaging: what role? Radiology 2010;257:24-39.

55. Schirin-Sokhan R, Winograd R, Tischendorf S, et al. Assessment of inflammatory and fibrotic stenoses in patients with Crohn's disease using contrast-enhanced ultrasound and computerized algorithm: a pilot study. Digestion 2011;83:263-268.

56. Lenze F, Wessling J, Bremer J, et al. Detection and differentiation of inflammatory versus fibromatous Crohn's disease strictures: prospective comparison of 18F-FDG-PET/CT, MR-enteroclysis, and transabdominal ultrasound versus endoscopic/histologic evaluation. Inflamm Bowel Dis 2012;18:2252-2260.

57. Quaia E, Migaleddu V, Baratella E, et al. The diagnostic value of small bowel wall vascularity after sulfur hexafluoride-filled microbubble injection in patients with Crohn's disease. Correlation with the therapeutic effectiveness of specific anti-inflammatory treatment. Eur J Radiol 2009;69:438-444.

58. Parente F, Molteni M, Marino B, et al. Are colonoscopy and bowel ultrasound useful for assessing response to short-term therapy and predicting disease outcome of moderate-to-severe forms of ulcerative colitis?: a prospective study. Am J Gastroenterol 2010;105:1150-1157.

59. Parente F, Molteni M, Marino B, et al. Bowel ultrasound and mucosal healing in ulcerative colitis. Dig Dis 2009;27:285-290.

60. Paredes JM, Ripollés T, Cortés X, et al. Contrast-enhanced ultrasonography: usefulness in the assessment of postoperative recurrence of Crohn's disease. J Crohns Colitis 2013;7:192-201.

61. Moreno N, Ripollés T, Paredes JM, et al. Usefulness of abdominal ultrasonography in the analysis of endoscopic activity in patients with Crohn's disease: changes following treatment with immunomodulators and/or anti-TNF antibodies. J Crohns Colitis 2014;8:1079-1087.

62. Kucharzik T, Wittig BM, Helwig U, et al(on behalf of the TRUST study group). Ultrasonography in useful for monitoring Crohn's disease - results from the German TRUST study. Clin Gastroenterol Hepatol 2016 [Epub ahead of print].

63. Ripollés T, Paredes Arquiola JM, Moreno-Osset E. Ultrasonography and transmural healing in Crohn's disease. Clin Gastroenterol Hepatol 2015;13:1549-1551.

64. Zorzi F, Stasi E, Bevivino G, et al. A sonographic lesion index for Crohn's disease helps monitor changes in transmural bowel damage during therapy. Clin Gastroenterol Hepatol 2014;12:2071-2077.

65. Castiglione F, Testa A, Rea M, et al. Transmural healing evaluated by bowel sonography in patients with Crohn's disease on maintenance treatment with biologics. Inflamm Bowel Dis 2013;19:1928-1934.

66. Rutgeerts P, Geboes K, Vantrappen G, Beyls J, Kerremans R, Hiele M. Predictability of the postoperative course of Crohn's disease. Gastroenterology 1990;99:956-963.

67. De Cruz P, Kamm MA, Hamilton AL, et al. Crohn's disease management after intestinal resection: a randomised trial. Lancet 2015;385:1406-1417.

68. Maconi G, Sampietro GM, Cristaldi M, et al. Preoperative characteristics and postoperative behavior of bowel wall on risk of recurrence after conservative surgery in Crohn's disease: a prospective study. Ann Surg 2001;233:345-352.

69. Castiglione F, de Sio I, Cozzolino A, et al. Bowel wall thickness at abdominal ultrasound and the one-year-risk of surgery in patients with Crohn's disease. Am J Gastroenterol 2004;99:1977-1983.

70. Rispo A, Bucci L, Pesce G, et al. Bowel sonography for the diagnosis and grading of postsurgical recurrence of Crohn's disease. Inflamm Bowel Dis 2006;12:486-490.

71. Kunihiro K, Hata J, Manabe N, et al. Predicting the need for surgery in Crohn's disease with contrast harmonic ultrasound. Scand J Gastroenterol 2007;42:577-585.

72. Castiglione F, Bucci L, Pesce G, et al. Oral contrast-enhanced sonography for the diagnosis and grading of postsurgical recurrence of Crohn's disease. Inflamm Bowel Dis 2008;14:1240-1245.

73. Pallotta N, Giovannone M, Pezzotti P, et al. Ultrasonographic detection and assessment of the severity of Crohn's disease recurrence after ileal resection. BMC Gastroenterol 2010;10:69.

74. Calabrese E, Zorzi F, Zuzzi S, et al. Development of a numerical index quantitating small bowel damage as detected by ultrasonography in Crohn's disease. J Crohns Colitis 2012;6:852-860.

75. Schwartz DA, Loftus EV Jr, Tremaine WJ, et al. The natural history of fistulizing Crohn's disease in Olmsted County, Minnesota. Gastroenterology 2002;122:875-880.

76. Tang LY, Rawsthorne P, Bernstein CN. Are perineal and luminal fistulas associated in Crohn's disease? A population-based study. Clin Gastroenterol Hepatol 2006;4:1130-1134.

77. Schwartz DA, Wiersema MJ, Dudiak KM, et al. A comparison of endoscopic ultrasound, magnetic resonance imaging, and exam under anesthesia for evaluation of Crohn's perianal fistulas. Gastroenterology 2001;121:1064-1072.

78. Siddiqui MR, Ashrafian H, Tozer P, et al. A diagnostic accuracy meta-analysis of endoanal ultrasound and MRI for perianal fistula assessment. Dis Colon Rectum 2012;55:576-585.

79. Maconi G, Ardizzone S, Greco S, Radice E, Bezzio C, Bianchi Porro G. Transperineal ultrasound in the detection of perianal and rectovaginal fistulae in Crohn's disease. Am J Gastroenterol 2007; 102:2214-2219.

80. Nevler A, Beer-Gabel M, Lebedyev A, et al. Transperineal ultrasonography in perianal Crohn's disease and recurrent cryptogenic fistula-in-ano. Colorectal Dis 2013;15:1011-1018.

81. Wedemeyer J, Kirchhoff T, Manns MP, Gebel MJ, Bleck JS. [Transcutaneous perianal ultrasound (PAUS) for the imaging 
of fistulas and abscesses in Crohn's disease]. Z Gastroenterol 2004;42:1315-1320.

82. Sudol-Szopińska I, Jakubowski W, Szczepkowski M, Sarti D. Usefulness of hydrogen peroxide enhancement in diagnosis of anal and ano-vaginal fistulas. Eur Radiol 2003;13:1080-1084.

83. West RL, Zimmerman DD, Dwarkasing S, et al. Prospective comparison of hydrogen peroxide-enhanced three-dimensional endoanal ultrasonography and endoanal magnetic resonance imaging of perianal fistulas. Dis Colon Rectum 2003;46:1407-1415.

84. Ludwig D, Wiener S, Brüning A, et al. Mesenteric blood flow is related to disease activity and risk of relapse in ulcerative colitis: a prospective follow up study. Gut 1999;45:546-552.

85. Homann N, Klarmann U, Fellermann K, et al. Mesenteric pulsatility index analysis predicts response to azathioprine in patients with Crohn's disease. Inflamm Bowel Dis 2005;11:126-132.

86. Siğirci A, Baysal T, Kutlu R, Aladağ M, Saraç K, Harputluoğlu H. Doppler sonography of the inferior and superior mesenteric arteries in ulcerative colitis. J Clin Ultrasound 2001;29:130-139.

87. Martínez MJ, Ripollés T, Paredes JM, Blanc E, Martí-Bonmatí L. Assessment of the extension and the inflammatory activity in Crohn's disease: comparison of ultrasound and MRI. Abdom Imaging 2009;34:141-148. 